\title{
Model Reduction Via Limited Frequency Interval Gramians
}

\author{
Abdul Ghafoor and Victor Sreeram, Senior Member, IEEE
}

\begin{abstract}
An improved frequency domain interval Gramianbased model reduction scheme for discrete time systems is presented. It is first shown that two of the main results presented in the model reduction method of [20] are incorrect. Improved methods which overcomes these shortcomings are then presented. Improved methods not only yields stable reduced-order models but also have easily computable frequency response error bounds. The method is further extended to 2-D separable denominator system approximation. The simulation results show the effectiveness of the proposed scheme.
\end{abstract}

Index Terms-Balanced truncation, interval Gramians, modelorder reduction, 2-D systems, frequency domain Gramians, frequency weightings.

\section{INTRODUCTION}

$\mathbf{T}$ HE balanced realization has been a significant contribution to system theory, especially, its application to model reduction known as balanced truncation [8], [13] which can preserve stability and give an explicit bound on frequency response error [3], [2].

Ideally, it is important that the reduction error between the original system and the reduced-order model is small for all frequencies. However, sometimes, the reduction error is more important over a certain frequency band than over other frequencies. Enns [3] has extended the balanced truncation [8] method to include frequency weightings. However, the Enns' method may yield unstable models for two-sided weightings. Gawronski and Juang [4] proposed a new balanced related model reduction method based on the concept of Gramians defined over a desired frequency interval for continuous time systems. A similar method also appears in [1]. However, the drawbacks of Gawronski and Juang's method are that it can yield unstable reduced-order models for stable original systems and there are no a prior bounds on the approximation error. Inspired by Wang et al. [18], Gugercin and Antoulas [5] modified the Gawronski and Juang's method [4] to obtain stable reduced-order models and error bounds. In [20], Wang and Zilouchian have extended the technique [1] (which is similar to

Manuscript received June 05, 2006; revised June 18, 2007 and November 09, 2007. First published March 07, 2008; current version published October 29, 2008. The work was supported by the Australian Research Council under the Discovery Grants Scheme. This paper was recommended by Associate Editor C. Hadjicostis.

A. Ghafoor was with the School of Electrical, Electronic and Computer Engineering, University of Western Australia, WA 6009, Australia. He is now with the National University of Sciences and Technology, Rawalpindi, Pakistan (e-mail: aghafoor@ee.uwa.edu.au; sreeram@ee.uwa.edu.au).

V. Sreeram is with the School of Electrical, Electronic and Computer Engineering, University of Western Australia, WA 6009, Australia (e-mail: sreeram@ee.uwa.edu.au).

Digital Object Identifier 10.1109/TCSI.2008.920092
[4]) for discrete-time sytems (Please also see [9] and [17] for corrections and authors' reply to the paper [20]). In the paper [20], the authors provide a proof of stability of reduced-order models and derivation of error bounds. In this paper we first show that both these results are incorrect. We then present some simple improvements to the method [20] to overcome these shortcomings. The improved methods have the following advantages: (i) guaranteed stability in the case of double-sided weighting and (ii) easily computable frequency response error bounds. Furthermore, this method is also extended to approximate 2-D separable denominator systems.

\section{WANG AND ZILOUCHIAN'S TECHNIQUE}

Consider a stable system $H_{1}(z)=C_{1}\left(z I-A_{1}\right)^{-1} B_{1}+D_{1}$, where $\left\{A_{1} \in \Re^{n \times n}, B_{1} \in \Re^{n \times q}, C_{1} \in \Re^{p \times n}, D_{1} \in \Re^{p \times q}\right\}$ is its minimal realization. The equivalent time and frequency domain controllability and observability Gramians

$$
\begin{aligned}
P_{c} & =\sum_{k=0}^{k=\infty} A_{1}^{k} B_{1} B_{1}^{T}\left(A_{1}^{T}\right)^{k} \\
& =\frac{1}{2 \pi} \int_{-\pi}^{\pi}\left(I-A_{1} e^{-j \omega}\right)^{-1} B_{1} B_{1}^{T}\left(I-A_{1}^{T} e^{j \omega}\right)^{-1} d \omega \\
Q_{o} & =\sum_{k=0}^{k=\infty}\left(A_{1}^{T}\right)^{k} C_{1}^{T} C_{1} A_{1}^{k} \\
& =\frac{1}{2 \pi} \int_{-\pi}^{\pi}\left(I-A_{1}^{T} e^{j \omega}\right)^{-1} C_{1}^{T} C_{1}\left(I-A_{1} e^{-j \omega}\right)^{-1} d \omega
\end{aligned}
$$

respectively, satisfy the following Lyapunov equations:

$$
\begin{gathered}
A_{1} P_{c} A_{1}^{T}-P_{c}=B_{1} B_{1}^{T} \\
A_{1}^{T} Q_{o} A_{1}-Q_{o}=C_{1}^{T} C_{1} .
\end{gathered}
$$

Definition 1: [20] The frequency domain controllability and observability Gramians are respectively defined by

$$
\begin{aligned}
P_{c f} & =\frac{1}{2 \pi} \int_{\delta \omega}\left(I-A_{1} e^{-j \omega}\right)^{-1} B_{1} B_{1}^{T}\left(I-A_{1}^{T} e^{j \omega}\right)^{-1} d \omega \\
Q_{o f} & =\frac{1}{2 \pi} \int_{\delta \omega}\left(I-A_{1}^{T} e^{j \omega}\right)^{-1} C_{1}^{T} C_{1}\left(I-A_{1} e^{-j \omega}\right)^{-1} d \omega
\end{aligned}
$$

where $\delta \omega=\left[\omega_{1}, \omega_{2}\right]$ is the frequency range of operation and $0 \leq \omega_{1}<\omega_{2} \leq \pi$. Note that due to symmetry of the Fourier transform, the integration is carried out over the intervals $\left[\omega_{1}, \omega_{2}\right]$ and $\left[-\omega_{2},-\omega_{1}\right]$ (see also [6]). This will ensure that the Gramians are always real.

The Gramians $P_{c f}$ and $Q_{\text {of }}$, respectively, satisfy

$$
\begin{aligned}
& A_{1} P_{c f} A_{1}^{T}-P_{c f}+X=0 \\
& A_{1}^{T} Q_{\text {of }} A_{1}-Q_{\text {of }}+Y=0
\end{aligned}
$$


where

$$
\begin{aligned}
& X=F B_{1} B_{1}^{T}+B_{1} B_{1}^{T} F^{*} \\
& Y=F^{*} C_{1}^{T} C_{1}+C_{1}^{T} C_{1} F \\
& F=-\frac{\omega_{2}-\omega_{1}}{4 \pi} I+\frac{1}{2 \pi} \int_{\delta \omega}\left(I-A_{1} e^{-j \omega}\right)^{-1} d \omega
\end{aligned}
$$

and $F^{*}$ is the conjugate transpose of $F$.

Remark 1: Note that for $\delta \omega=[0, \pi]$, the Gramians $P_{c}=P_{c f}$ and $Q_{o}=Q_{\text {of }}$.

Simultaneously diagonalizing the frequency domain Gramians $P_{c f}$ and $Q_{\text {of }}$, we get

$$
T^{T} Q_{\text {of }} T=T^{-1} P_{c f} T^{-T}=\operatorname{diag}\left\{\sigma_{1}, \sigma_{2}, \ldots, \sigma_{n}\right\}
$$

where $\sigma_{i} \geq \sigma_{i+1}, i=1,2, \ldots, n-1$ and $\sigma_{r}>\sigma_{r+1}$. Transforming and partitioning the original system, we get

$$
\begin{aligned}
& \hat{A}=T^{-1} A_{1} T=\left[\begin{array}{ll}
A_{11} & A_{12} \\
A_{21} & A_{22}
\end{array}\right] \\
& \hat{B}=T^{-1} B_{1}=\left[\begin{array}{l}
B_{11} \\
B_{12}
\end{array}\right] \\
& \hat{C}=C_{1} T=\left[\begin{array}{ll}
C_{11} & C_{12}
\end{array}\right]
\end{aligned}
$$

where $A_{11} \in \Re^{r \times r}$. The reduced-order model is given by $H_{r_{1}}(z)=C_{11}\left(z I-A_{11}\right)^{-1} B_{11}+D_{1}$.

Theorem 2: ([20, Th. 5.2]) If the system $\left(A_{1}, B_{1}, C_{1}\right)$ is asymptotically stable, controllable, and observable, then every reduced-order subsystem is also asymptotically stable.

Theorem 2: ([20, Th. 5.6]) The error bound for the frequency weighted model reduction technique is

$\left\|C_{1}\left(z I-A_{1}\right)^{-1} B_{1}-C_{11}\left(z I-A_{11}\right)^{-1} B_{11}\right\|_{\infty} \leq \frac{2}{\beta} \sum_{k=r+1}^{n} \sigma_{k}$

where $\beta=2 \sigma_{\min }(\operatorname{Re}(F))$.

\section{MAIN RESUlts}

In [20], it is claimed that if the matrices $P_{c f}$ and $Q_{\text {of }}$ are diagonal and positive definite then the matrices $X$ and $Y$ [see (3)-(4)]

$$
\begin{aligned}
& X=P_{c f}-A_{1} P_{c f} A_{1}^{T} \\
& Y=Q_{\text {of }}-A_{1}^{T} Q_{\text {of }} A_{1}
\end{aligned}
$$

are also positive definite, for stable $A_{1}$ matrix. The proofs of [20, Th. 5.2 and 5.6] are based on this claim. Here we first show by an example that this claim is not true.

Example 1: Let $P=\left[\begin{array}{cc}1 & 0 \\ 0 & 0.2\end{array}\right]$ and $A=\left[\begin{array}{cc}-0.5 & 0.4 \\ 0.4 & 0.4\end{array}\right]$ with eigenvalues -0.6521 and 0.5521 . Then the matrix

$$
\mathrm{APA}^{T}-P=\left[\begin{array}{ll}
-0.718 & -0.168 \\
-0.168 & -0.008
\end{array}\right]
$$

is indefinite having eigenvalues -0.7557 and 0.0297 .

See [7], for more information on indefiniteness of this type of matrices.
Since this claim is essential to guarantee the stability of reduced-order models and the existence of the frequency response error bound, the method [20] may produce unstable models and error bound does not exist.

Note also that error bound (if it exists) can only be given if the stability of the reduced-order model is guaranteed. In the next subsection, we show how their method [20] can be modified to: (i) guarantee the stability of reduced-order models and (ii) to derive frequency response error bounds.

\section{A. Stability Preserving 1-D Approximation Using Frequency Domain Interval Gramians - I}

Since the matrices $X$ and $Y$ are real symmetric, there exist orthogonal matrices $U, V$ and diagonal matrices $S, H$ such that

$$
\begin{aligned}
& X=\mathrm{USU}^{T} \\
& Y=V H V^{T}
\end{aligned}
$$

where

$$
\begin{aligned}
S= & \operatorname{diag}\left(s_{1}, s_{2}, \ldots, s_{n}\right), \\
H= & \operatorname{diag}\left(h_{1}, h_{2}, \ldots, h_{n}\right) \\
& \left|s_{1}\right| \geq\left|s_{2}\right| \geq \cdots \geq\left|s_{n}\right| \geq 0,\left|h_{1}\right| \geq\left|h_{2}\right| \geq \cdots \geq\left|h_{n}\right| \geq 0 .
\end{aligned}
$$

Let the new controllability and observability frequency domain interval Gramians, respectively, be defined as follows:

$$
\begin{aligned}
& A_{1} \bar{P}_{c f} A_{1}^{T}-\bar{P}_{c f}+\bar{B}_{1} \bar{B}_{1}^{T}=0 \\
& A_{1}^{T} \bar{Q}_{\text {of }} A_{1}-\bar{Q}_{c f}+\bar{C}_{1}^{T} \bar{C}_{1}=0
\end{aligned}
$$

where the new fictitious input and output matrices are defined, respectively, as $\bar{B}_{1}:=U|S|^{(1) /(2)}$ and $\bar{C}_{1}:=|H|^{(1) /(2)} V^{T}$.

Lemma 1: Assume that

$$
\begin{aligned}
& \operatorname{rank}\left[\begin{array}{cc}
\bar{B}_{1} & B_{1}
\end{array}\right]=\operatorname{rank}\left[\bar{B}_{1}\right] \\
& \operatorname{rank}\left[\begin{array}{l}
C_{1} \\
\bar{C}_{1}
\end{array}\right]=\operatorname{rank}\left[\bar{C}_{1}\right]
\end{aligned}
$$

then $B_{1}=\bar{B}_{1} K_{1}$ and $C_{1}=L_{1} \bar{C}_{1}$ where

$$
\begin{aligned}
& K_{1}=\operatorname{diag}\left(\left|s_{1}\right|^{-\frac{1}{2}},\left|s_{2}\right|^{-\frac{1}{2}}, \ldots,\left|s_{i_{1}}\right|^{-\frac{1}{2}}, 0, \ldots, 0\right) U^{T} B_{1} \\
& L_{1}=C_{1} V \operatorname{diag}\left(\left|h_{1}\right|^{-\frac{1}{2}},\left|h_{2}\right|^{-\frac{1}{2}}, \ldots,\left|h_{j_{1}}\right|^{-\frac{1}{2}}, 0, \ldots, 0\right) \\
& \operatorname{rank}[X]=i_{1} \text { and } \operatorname{rank}[Y]=j_{1} . \\
& \quad \text { Proof: Similar to that in }[18] .
\end{aligned}
$$

Remark 2: It is shown in [18] that (13) and (14) are almost always true.

Theorem 3: The realization $\left\{A_{1}, \bar{B}_{1}, \bar{C}_{1}\right\}$ is stable and minimal.

Proof: The proof follows from the stability and minimality of the realization $\left\{A_{1}, B_{1}, C_{1}\right\}$.

Let $T_{1}$ be the transformation obtained by simultaneously diagonalizing the frequency domain interval Gramians $\bar{P}_{c f}$ and $\bar{Q}_{c f}$

$$
T_{1}^{T} \bar{Q}_{\text {of }} T_{1}=T_{1}^{-1} \bar{P}_{c f} T_{1}^{-T}=\operatorname{diag}\left\{\sigma_{1}, \sigma_{2}, \ldots, \sigma_{n}\right\}
$$

where $\sigma_{i} \geq \sigma_{i+1}, i=1,2, \ldots, n-1$ and $\sigma_{r}>\sigma_{r+1}$. 
The reduced-order model $\left\{A_{1 b 1}, B_{1 b 1}, C_{1 b 1}, D_{1}\right\}$ is obtained by transforming and partitioning the original system realization

$$
\left[\begin{array}{c|c}
T_{1}^{-1} A_{1} T_{1} & T_{1}^{-1} B_{1} \\
\hline C_{1} T_{1} & D_{1}
\end{array}\right]\left[\begin{array}{cc|c}
A_{1 b 1} & A_{1 b 2} & B_{1 b 1} \\
A_{1 b 3} & A_{1 b 4} & B_{1 b 2} \\
\hline C_{1 b 1} & C_{1 b 2} & D_{1}
\end{array}\right] .
$$

Algorithm 1: Given $H_{1}(z)$ and desired frequency range $\left[\omega_{1}, \omega_{2}\right]$ for approximation, reduced-order model is obtained using the following steps:

i) Use (5)-(6) to compute $X$ and $Y$.

ii) Use (9)-(10) to decompose $X$ and $Y$, respectively, to obtain $\bar{B}_{1}=U|S|^{(1) /(2)}$ and $\bar{C}_{1}=|H|^{(1) /(2)} V^{T}$.

iii) Solve the (11)-(12) to compute $\bar{P}_{c f}$ and $\bar{Q}_{\text {of }}$.

iv) Find the transformation $T_{1}$ to satisfy the (15).

v) Compute and partition the balanced realization [(16)].

vi) Obtain the reduced-order model: $\left\{A_{1 b 1}, B_{1 b 1}\right.$, $\left.C_{1 b 1}, D_{1}\right\}$.

Theorem 4: The reduced-order models obtained using the Algorithm 1 are stable.

Proof: The proof follows immediately from the proof of stability of the unweighted approximation [13] and is, therefore, omitted.

Theorem 5: Let the reduced-order models be obtained by the algorithm, then frequency response error is bounded by

$$
\left\|H_{1}(z)-H_{1 r}(z)\right\|_{\infty} \leq 2\left\|L_{1}\right\|\left\|K_{1}\right\| \sum_{i=r+1}^{n} \sigma_{i}
$$

Proof: Partitioning $\bar{B}_{1}=\left[\begin{array}{l}\bar{B}_{11} \\ \bar{B}_{12}\end{array}\right], \bar{C}_{1}=\left[\begin{array}{ll}\bar{C}_{11} & \bar{C}_{12}\end{array}\right]$, substituting $B_{1 b 1}=\bar{B}_{11} K_{1}, C_{1 b 1}=L_{1} \bar{C}_{11}$ and using Lemma 1, we can write

$$
\begin{aligned}
& \left\|H_{1}(z)-H_{1 r}(z)\right\|_{\infty} \\
& =\left\|C_{1}\left(z I-A_{1}\right)^{-1} B_{1}-C_{1 b 1}\left(z I-A_{1 b 1}\right)^{-1} B_{1 b 1}\right\|_{\infty} \\
& =\left\|L_{1}\left(\bar{C}_{1}\left(z I-A_{1}\right)^{-1} \bar{B}_{1}-\bar{C}_{11}\left(z I-A_{1 b 1}\right)^{-1} \bar{B}_{11}\right) K_{1}\right\|_{\infty} \\
& \leq 2\left\|L_{1}\right\|\left\|K_{1}\right\| \sum_{i=r+1}^{n} \sigma_{i}
\end{aligned}
$$

where from [2], we have

$\left\|\bar{C}_{1}\left(z I-A_{1}\right)^{-1} \bar{B}_{1}-\bar{C}_{11}\left(z I-A_{1 b 1}\right)^{-1} \bar{B}_{11}\right\|_{\infty} \leq 2 \sum_{i=r+1}^{n} \sigma_{i}$.

Remark 3: Note that, when $X \geq 0$ and $Y \geq 0$, the proposed method is equal to Wang and Zilouchian's method. However, in general, the matrices $X$ and $Y$ are indefinite. The frequency domain interval Gramians satisfy $P_{c f} \leq \bar{P}_{c f}$ and $Q_{\text {of }} \leq \bar{Q}_{\text {of }}$. In order to reduce the distances between the Gramians, $\overline{\bar{P}}_{c f}-P_{c f}$ and $\bar{P}_{\text {of }}-P_{\text {of }}$, inspired by [16] we propose another improved model reduction scheme.

\section{B. Stability Preserving 1-D Approximation Using Frequency Domain Interval Gramians - II}

In this technique, the new controllability and observability Gramians $\hat{P}_{c f}$ and $\hat{P}_{\text {of }}$, respectively obtained as the solutions to
Lyapunov equations

$$
\begin{aligned}
& A_{1} \hat{P}_{c f} A_{1}^{T}-\hat{P}_{c f}+\hat{B}_{1} \hat{B}_{1}^{T}=0 \\
& A_{1}^{T} \hat{P}_{\text {of }} A_{1}^{T}-\hat{P}_{\text {of }}+\hat{C}_{1}^{T} \hat{C}_{1}=0
\end{aligned}
$$

are simultaneously diagonalized

$$
\hat{T}_{1}^{T} \hat{P}_{\mathrm{of}} \hat{T}_{1}=\hat{T}_{1}^{-1} \hat{P}_{c f} \hat{T}_{1}^{-T}=\operatorname{diag}\left\{\hat{\sigma}_{1}, \hat{\sigma}_{2}, \ldots, \hat{\sigma}_{n}\right\}
$$

$\hat{\sigma}_{i} \geq \hat{\sigma}_{i+1}, i=1,2, \ldots, n-1$ and $\hat{\sigma}_{r}>\hat{\sigma}_{r+1}$. The new fictitious matrices $\hat{B}_{1}$ and $\hat{C}_{1}$ in the above Lyapunov equations are defined as $\hat{B}_{1}=\hat{U}_{1} \hat{S}_{1}^{1 / 2}$ and $\hat{C}_{1}=\hat{R}_{1}^{1 / 2} \hat{V}_{1}^{T}$, respectively. The terms $\hat{U}_{1}, \hat{S}_{1}, \hat{V}_{1}$, and $\hat{R}_{1}$ are obtained from the orthogonal eigendecomposition of symmetric matrices

$$
\begin{aligned}
& X=\left[\begin{array}{ll}
\hat{U}_{1} & \hat{U}_{2}
\end{array}\right]\left[\begin{array}{cc}
\hat{S}_{1} & 0 \\
0 & \hat{S}_{2}
\end{array}\right]\left[\begin{array}{c}
\hat{U}_{1}^{T} \\
\hat{U}_{2}^{T}
\end{array}\right] \\
& Y=\left[\begin{array}{ll}
\hat{V}_{1} & \hat{V}_{2}
\end{array}\right]\left[\begin{array}{cc}
\hat{R}_{1} & 0 \\
0 & \hat{R}_{2}
\end{array}\right]\left[\begin{array}{c}
\hat{V}_{1}^{T} \\
\hat{V}_{2}^{T}
\end{array}\right]
\end{aligned}
$$

where $\left[\begin{array}{cc}\hat{S}_{1} & 0 \\ 0 & \hat{S}_{2}\end{array}\right]=\operatorname{diag}\left(s_{1}, s_{2}, \ldots, s_{n}\right),\left[\begin{array}{cc}\hat{R}_{1} & 0 \\ 0 & \hat{R}_{2}\end{array}\right]=$ $\operatorname{diag}\left(r_{1}, r_{2}, \ldots, r_{n}\right), s_{1} \geq s_{2} \geq \cdots \geq s_{n}, r_{1} \geq$ $r_{2} \geq \cdots \geq r_{n}, \hat{S}_{1}=\operatorname{diag}\left(s_{1}, s_{2}, \ldots, s_{k}\right), \hat{R}_{1}=$ $\operatorname{diag}\left(r_{1}, r_{2}, \ldots, r_{k}\right), s_{1} \geq s_{2} \geq \cdots \geq s_{k}>0, r_{1} \geq$ $r_{2} \geq \cdots \geq r_{k}>0$.

Note that the realization $\left\{A_{1}, \hat{B}_{1}, \hat{C}_{1}\right\}$ is stable and minimal.

The reduced-order model $\left\{\hat{A}_{1 b 1}, \hat{B}_{1 b 1}, \hat{C}_{1 b 1}, D_{1}\right\}$ is obtained by transforming, partitioning, and truncating the original system realization

$$
\left[\begin{array}{c|c}
\hat{T}_{1}^{-1} A_{1} \hat{T}_{1} & \hat{T}_{1}^{-1} B_{1} \\
\hline C_{1} \hat{T}_{1} & D_{1}
\end{array}\right]\left[\begin{array}{c|cc}
\hat{A}_{1 b 1} & \hat{A}_{1 b 2} & \hat{B}_{1 b 1} \\
\hat{A}_{1 b 3} & \hat{A}_{1 b 4} & \hat{B}_{1 b 2} \\
\hline \hat{C}_{1 b 1} & \hat{C}_{1 b 2} & D_{1}
\end{array}\right] .
$$

Algorithm 2: Given $H_{1}(z)$ and desired frequency range $\left[\omega_{1}, \omega_{2}\right]$ for approximation, reduced-order model is obtained using the following steps:

i) Use (5)-(6) to compute $X$ and $Y$.

ii) Use (20)-(21) to decompose $X$ and $Y$, respectively, to obtain $\hat{B}_{1}=\hat{U}_{1} \hat{S}_{1}^{1 / 2}$ and $\hat{C}_{1}=\hat{R}_{1}^{1 / 2} \hat{V}_{1}^{T}$.

iii) Solve (17)-(18) to compute $\hat{P}_{c f}$ and $\hat{Q}_{\text {of }}$.

iv) Find the transformation $\hat{T}_{1}$ to satisfy (19).

v) Compute the balanced realization [(22)].

vi) Obtain the reduced-order $\left\{\hat{A}_{1 b 1}, \hat{B}_{1 b 1}, \hat{C}_{1 b 1}, D_{1}\right\}$.

model:

Theorem 6: The reduced-order models obtained using Algorithm 2 are stable.

Proof: The proof follows immediately from the proof of stability of the unweighted approximation [13] and is, therefore, omitted.

Remark 4: The frequency domain interval Gramians satisfy $P_{c f} \leq \hat{P}_{c f} \leq \bar{P}_{c f}$ and $Q_{\text {of }} \leq \hat{P}_{c f} \leq \bar{Q}_{\text {of }}$. The equality holds when $X \geq 0$ and $Y \geq 0$.

Remark 5: The frequency response error bound (similar to Theorem 5) holds subject to fulfillment of the following rank conditions:

$$
\begin{aligned}
& \operatorname{rank}\left[\begin{array}{l}
\hat{B}_{1} \\
B_{1}
\end{array}\right]=\operatorname{rank}\left[\hat{B}_{1}\right] \\
& \operatorname{rank}\left[\begin{array}{l}
C_{1} \\
\hat{C}_{1}
\end{array}\right]=\operatorname{rank}\left[\hat{C}_{1}\right] .
\end{aligned}
$$




\section{2-D Approximation Using Frequency Domain Gramians}

Let $H\left(z_{1}, z_{2}\right) \in \Re^{p \times q}$ be a stable, minimal, and separable denominator transfer function matrix of a system of order $(n, m)$. The Roesser state-space model [10] to describe $H\left(z_{1}, z_{2}\right)$ can be written as

$$
H\left(z_{1}, z_{2}\right)=C\left(z_{1} I_{n} \oplus z_{2} I_{m}-A\right)^{-1} B+D_{*}
$$

where

$$
A=\left[\begin{array}{cc}
A_{1} & A_{*} \\
0 & A_{2}
\end{array}\right], B=\left[\begin{array}{c}
B_{*} \\
B_{2}
\end{array}\right], C=\left[\begin{array}{c}
C_{1}^{T} \\
C_{*}^{T}
\end{array}\right]^{T}
$$

the symbol $\oplus$ denotes the direct sum, $I$ is the identity matrix, $A \in \Re^{(n+m) \times(n+m)}, B \in \Re^{(n+m) \times q}, C \in$ $\Re^{p \times(n+m)}$, and $D_{*} \in \Re^{p \times q}$.

Let the minimal rank decomposition (see [21] for more details) of the Roesser state-space realization [(23)] be written as follows:

$$
\left[\begin{array}{ll}
A_{*} & B_{*} \\
C_{*} & D_{*}
\end{array}\right]=\left[\begin{array}{l}
B_{1} \\
D_{1}
\end{array}\right]\left[\begin{array}{ll}
C_{2} & D_{2}
\end{array}\right]
$$

then we can write $H\left(z_{1}, z_{2}\right)=H_{1}\left(z_{1}\right) H_{2}\left(z_{2}\right)$.

Similarly, the minimal rank decomposition for (23)

$$
\left[\begin{array}{ll}
A_{*} & B_{*} \\
C_{*} & D_{*}
\end{array}\right]=\left[\begin{array}{c}
B_{2} \\
D_{2}
\end{array}\right]\left[\begin{array}{ll}
C_{1} & D_{1}
\end{array}\right]
$$

allows us to write $H\left(z_{1}, z_{2}\right)=H_{2}\left(z_{2}\right) H_{1}\left(z_{1}\right)$ where

$$
\begin{aligned}
& H_{1}\left(z_{1}\right)=C_{1}\left(z_{1} I-A_{1}\right)^{-1} B_{1}+D_{1} \\
& H_{2}\left(z_{2}\right)=C_{2}\left(z_{2} I-A_{2}\right)^{-1} B_{2}+D_{2} .
\end{aligned}
$$

Algorithm 3: Given $H\left(z_{1}, z_{2}\right)$ and the desired frequency ranges $\left(\left[\omega_{1}, \omega_{2}\right]\right.$ and $\left.\left[\omega_{3}, \omega_{4}\right]\right)$ for approximation, reduced-order model is obtained using the following steps.

i) Decompose $H\left(z_{1}, z_{2}\right)$ into $H_{1}\left(z_{1}\right)$ and $H_{2}\left(z_{2}\right)$ as in (24)-(25).

ii) Use Algorithm 1 or 2 to find 1-D reduced-order models $\left\{A_{1 b 1}, B_{1 b 1}, C_{1 b 1}, D_{1}\right\}$ and $\left\{A_{2 b 1}, B_{2 b 1}, C_{2 b 1}, D_{2}\right\}$, respectively.

iii) The 2-D reduced-order model is

$$
\left[\begin{array}{c|c}
A_{r} & B_{r} \\
\hline C_{r} & D_{r}
\end{array}\right]=\left[\begin{array}{cc|c}
A_{1 b 1} & B_{1 b 1} C_{2 b 1} & B_{1 b 1} D_{2} \\
0 & A_{2 b 1} & B_{2 b 1} \\
\hline C_{1 b 1} & D_{1} C_{2 b 1} & D_{1} D_{2}
\end{array}\right]
$$

where

$$
\begin{aligned}
& A_{r} \in \Re^{\left(n_{r}+m_{r}\right) \times\left(n_{r}+m_{r}\right)} \\
& B_{r} \in \Re^{\left(n_{r}+m_{r}\right) \times q} \\
& C_{r} \in \Re^{p \times\left(n_{r}+m_{r}\right)} \\
& D_{r} \in \Re^{p \times q}, n_{r} \leq n \text { and } m_{r} \leq m .
\end{aligned}
$$

Theorem 7: Let $H\left(z_{1}, z_{2}\right)$ be a stable original system and $H_{r}\left(z_{1}, z_{2}\right)$ be the reduced-order model obtained by Algorithm 3 , then

i) The reduced-order model is stable. ii) The frequency response error is bounded by

$$
\begin{aligned}
& \left\|H\left(z_{1}, z_{2}\right)-H_{r}\left(z_{1}, z_{2}\right)\right\|_{\infty} \\
& \leq \quad\left(\left\|D_{1}\right\|+2\left\|L_{1}\right\|\left\|K_{1}\right\| \sum_{i=1}^{n} \sigma_{i}\right) \\
& \quad \times 2\left\|L_{2}\right\|\left\|K_{2}\right\| \sum_{i=m_{r}+1}^{m} \varphi_{i} \\
& \quad+\left(\left\|D_{2}\right\|+2\left\|L_{2}\right\|\left\|K_{2}\right\| \sum_{i=1}^{m_{r}} \varphi_{i}\right) \\
& \quad \times 2\left\|L_{1}\right\|\left\|K_{1}\right\| \sum_{i=n_{r}+1}^{n} \sigma_{i}
\end{aligned}
$$

alternatively

$$
\begin{aligned}
& \left\|H\left(z_{1}, z_{2}\right)-H_{r}\left(z_{1}, z_{2}\right)\right\|_{\infty} \\
& \leq\left(\left\|D_{1}\right\|+2\left\|L_{1}\right\|\left\|K_{1}\right\| \sum_{i=1}^{n_{r}} \sigma_{i}\right) \\
& \quad \times 2\left\|L_{2}\right\|\left\|K_{2}\right\| \sum_{i=m_{r}+1}^{m} \varphi_{i} \\
& \quad+\left(\left\|D_{2}\right\|+2\left\|L_{2}\right\|\left\|K_{2}\right\| \sum_{i=1}^{m} \varphi_{i}\right) \\
& \quad \times 2\left\|L_{1}\right\|\left\|K_{1}\right\| \sum_{i=n_{r}+1}^{n} \sigma_{i}
\end{aligned}
$$

where $\sigma_{i}$ and $\varphi_{i}$ are the frequency weighted Hankel singular values taken from (15) for horizontal and vertical systems, respectively.

Proof:

i) This follows immediately from the stability of the 1-D reduced-order models.

ii) For the error bound, note that

iii)

$$
\begin{aligned}
&\left\|H_{1}\left(z_{1}\right)\right\|_{\infty} \leq\left\|D_{1}\right\|+2\left\|L_{1}\right\|\left\|K_{1}\right\| \sum_{i=1}^{n} \sigma_{i} \\
&\left\|H_{2}\left(z_{2}\right)\right\|_{\infty} \leq\left\|D_{2}\right\|+2\left\|L_{2}\right\|\left\|K_{2}\right\| \sum_{i=1}^{m} \varphi_{i} \\
&\left\|H_{1 r}\left(z_{1}\right)\right\|_{\infty} \leq\left\|D_{1}\right\|+2\left\|L_{1}\right\|\left\|K_{1}\right\| \sum_{i=1}^{n_{r}} \sigma_{i} \\
&\left\|H_{2 r}\left(z_{2}\right)\right\|_{\infty} \leq\left\|D_{2}\right\|+2\left\|L_{2}\right\|\left\|K_{2}\right\| \sum_{i=1}^{m_{r}} \varphi_{i} \\
&\left\|H_{1}\left(z_{1}\right)-H_{1 r}\left(z_{1}\right)\right\|_{\infty} \leq 2\left\|L_{1}\right\|\left\|K_{1}\right\| \sum_{i=1+n_{r}}^{n} \sigma_{i} \\
&\left\|H_{2}\left(z_{2}\right)-H_{2 r}\left(z_{2}\right)\right\|_{\infty} \leq 2\left\|L_{2}\right\|\left\|K_{2}\right\| \sum_{i=1+m_{r}}^{m} \varphi_{i} .
\end{aligned}
$$

Furthermore

$$
\begin{aligned}
&\left\|H\left(z_{1}, z_{2}\right)-H_{r}\left(z_{1}, z_{2}\right)\right\|_{\infty} \\
&=\left\|H_{1}\left(z_{1}\right) H_{2}\left(z_{2}\right)-H_{1 r}\left(z_{1}\right) H_{2 r}\left(z_{2}\right)\right\|_{\infty} \\
&= \| H_{1}\left(z_{1}\right) H_{2}\left(z_{2}\right)-H_{1}\left(z_{1}\right) H_{2 r}\left(z_{2}\right) \\
&+H_{1}\left(z_{1}\right) H_{2 r}\left(z_{2}\right)-H_{1 r}\left(z_{1}\right) H_{2 r}\left(z_{2}\right) \|_{\infty} \\
& \leq\left\|H_{1}\left(z_{1}\right)\right\|_{\infty}\left\|H_{2}\left(z_{2}\right)-H_{2 r}\left(z_{2}\right)\right\|_{\infty} \\
&+\left\|H_{1}\left(z_{1}\right)-H_{1 r}\left(z_{1}\right)\right\|_{\infty}\left\|H_{2 r}\left(z_{2}\right)\right\|_{\infty} .
\end{aligned}
$$


Similarly

$$
\begin{aligned}
& \left\|H\left(z_{1}, z_{2}\right)-H_{r}\left(z_{1}, z_{2}\right)\right\|_{\infty} \\
& \quad \leq\left\|H_{1 r}\left(z_{1}\right)\right\|_{\infty}\left\|H_{2}\left(z_{2}\right)-H_{2 r}\left(z_{2}\right)\right\|_{\infty} \\
& \quad+\left\|H_{1}\left(z_{1}\right)-H_{1 r}\left(z_{1}\right)\right\|_{\infty}\left\|H_{2}\left(z_{2}\right)\right\|_{\infty} .
\end{aligned}
$$

The result follows.

\section{NUMERICAL RESULTS}

Example 2: This example is studied in [19, (Example 5.1)]. The stable original system is given by

$$
H(z)=\frac{10^{-3}\left(3.315 z^{3}-4.9695 z^{2}+2.1668 z-0.24002\right)}{z^{4}-3.7035 z^{3}+5.1957 z^{2}-3.2718 z+0.77986 .}
$$

The frequency weighted balanced realization obtained using Wang and Zilouchian's method [20], [19] in the frequency interval $0.25 \pi$ to $0.75 \pi$ is given by

$$
\begin{aligned}
& H(z)= \\
& {\left[\begin{array}{cccc|c}
0.4448 & -0.7953 & -0.0185 & 0.0044 & 0.0115 \\
0.7953 & 1.7479 & -0.3168 & -0.0141 & 0.0570 \\
0.0185 & -0.3168 & 0.7162 & 0.0613 & -0.0142 \\
0.0044 & 0.0141 & -0.0613 & 0.7947 & 0.0006 \\
\hline-0.0115 & 0.0570 & -0.0142 & -0.0006 & 0
\end{array}\right] .}
\end{aligned}
$$

The second-order reduced-order model obtained via partitioning and truncating has system poles at $1.0963+0.4561 i$ and $1.0963-0.4561 i$. The third-order reduced-order model obtained via partitioning and truncating has system poles at $1.0522,0.9283+0.2547 i$ and $0.9283-0.2547 i$. Clearly, the second- and third-order models obtained by Wang and Zilouchian's method [20], [19] are unstable, where as our proposed scheme produces guaranteed stable reduced-order models.

Example 3: Consider a six-order Elliptic digital filter with passband between $[0.1 \pi, 0.5 \pi]$, peak to peak ripples $0.1 \mathrm{~dB}$ and minimum stopband attenuation of $30 \mathrm{~dB}$. The filter transfer function is given by

$$
H(z)=\frac{0.1899 z^{6}-0.1072 z^{5}-0.3443 z^{4}+0.0000 z^{3}}{+0.3443 z^{2}+0.1072 z-0.1899}
$$

Fig. 2 shows the frequency response errors, $\sigma\left[H(z)-H_{r}(z)\right]$, where $H_{r}(z)$ represent the third-order reduced-order models produced by the proposed Algorithms 1 and 2, Wang and Zilouchian's method [20] and the balanced truncation [13]. A close up view in the desired frequency interval is given in Fig. 3. Note that, in the close up view (Fig. 3), the frequency response error obtained using unweighted balanced truncation method [13] is not shown because of large value. It is clear from the figure that the proposed technique compares well with other techniques [13] as shown in the matrix at the bottom of the next page.

Fig. 1 shows the frequency responses for third-order reducedorder models produced by the proposed Algorithms (1 and 2), Wang and Zilouchian's method [20], balanced truncation [13]

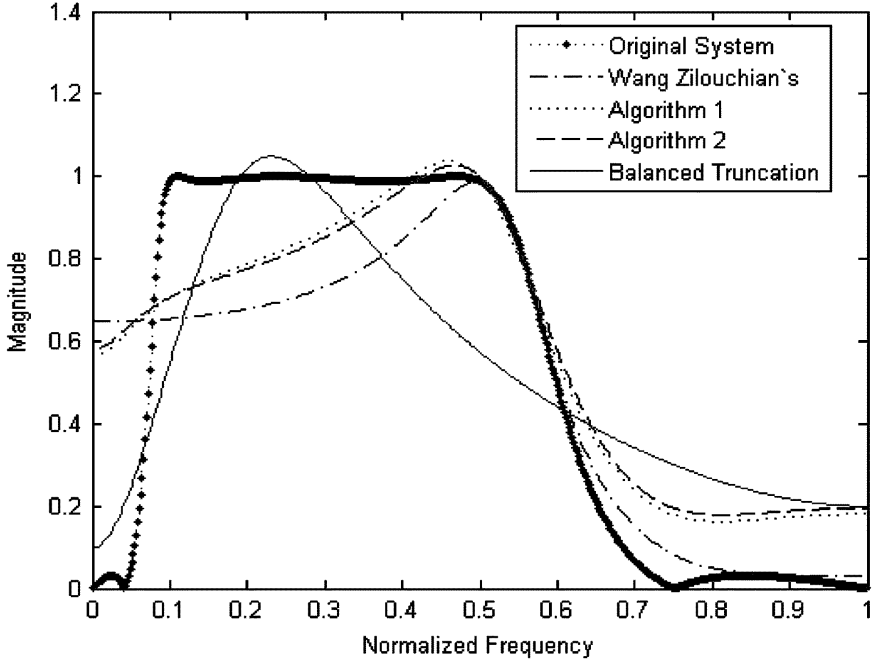

Fig. 1. Frequency response comparison.

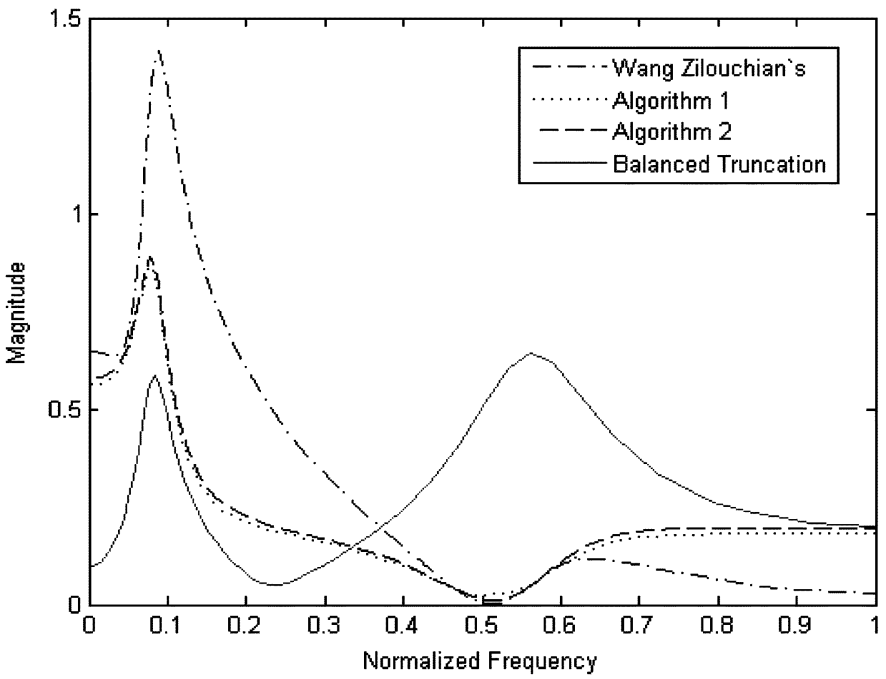

Fig. 2. Frequency response error comparison.

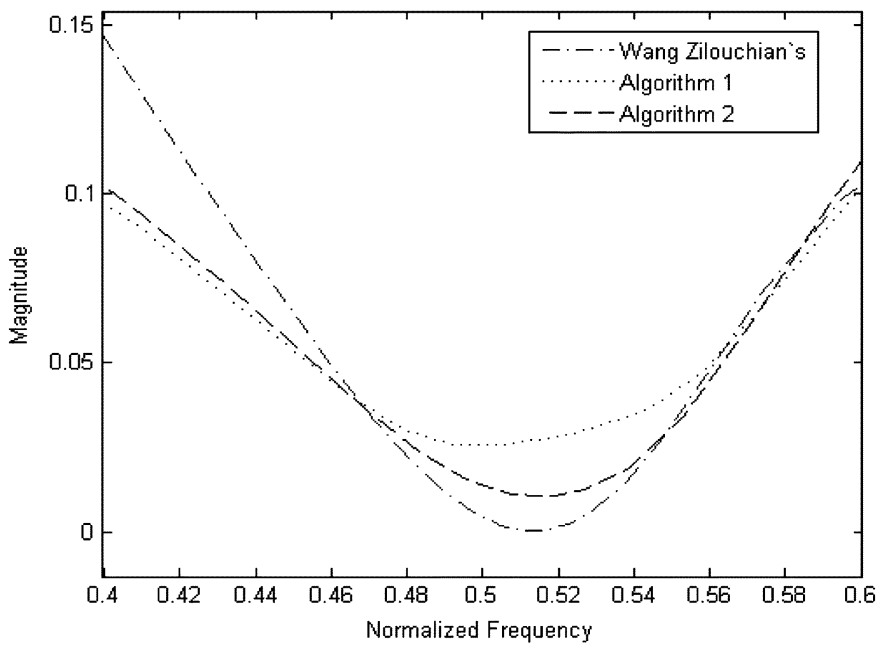

Fig. 3. Frequency response error comparison.

and the original system in the interval 0 to $\pi$. Note that the frequency interval for computing the Gramians in the proposed 


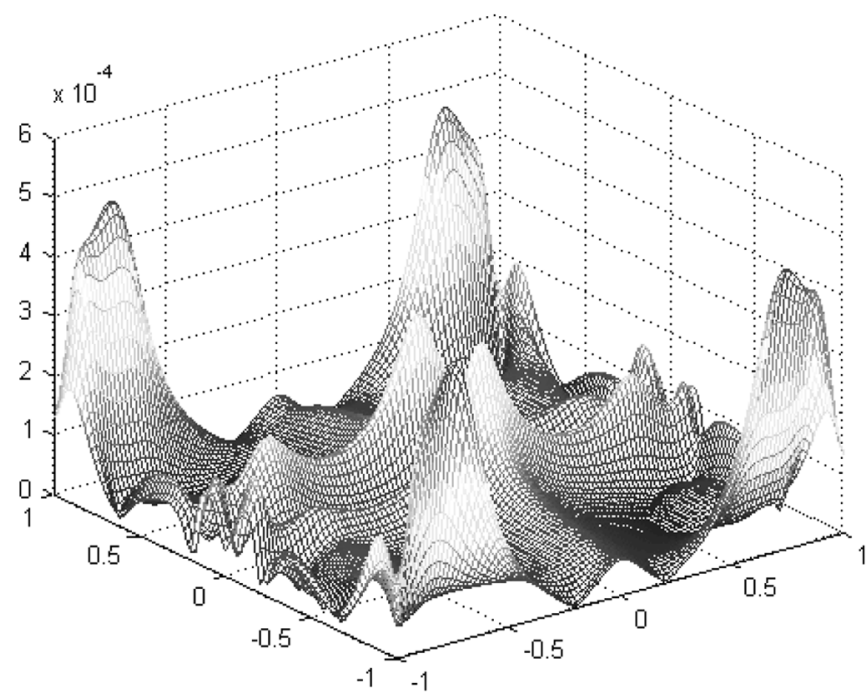

Fig. 4. Original system.

Algorithms (1 and 2) and Wang and Zilouchian's method is $0.4 \pi$ to $0.6 \pi$, where as frequency interval for computing the Gramians in the balanced truncation [13] is 0 to $\pi$.

Example 4: Consider the 2-D separable denominator $(6,6)$ order system studied in [14]. A state space representation of the original system is given in (26). Fig. 4 shows the frequency response of the original system. Fig. 5 shows the frequency response of a $(3,3)$-order reduced-order model obtained using Algorithm 3 (based on Algorithm 2) in the frequency interval 0 to $0.3 \pi$. Fig. 6 shows the frequency response of a $(4,4)$-order reduced-order model obtained using Algorithm 3 (based on Algorithm 2) in the frequency interval 0.6 to $\pi$. Comparing the original system and the reduced-order model frequency responses, it is clear that the proposed technique produces a better approximation in the desired frequency interval than over the entire frequency band.

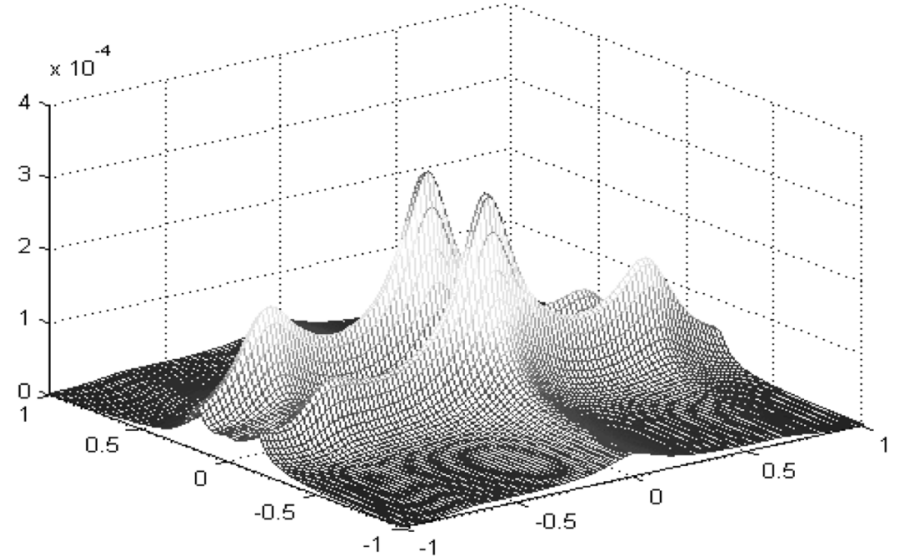

Fig. 5. Reduced-order System.

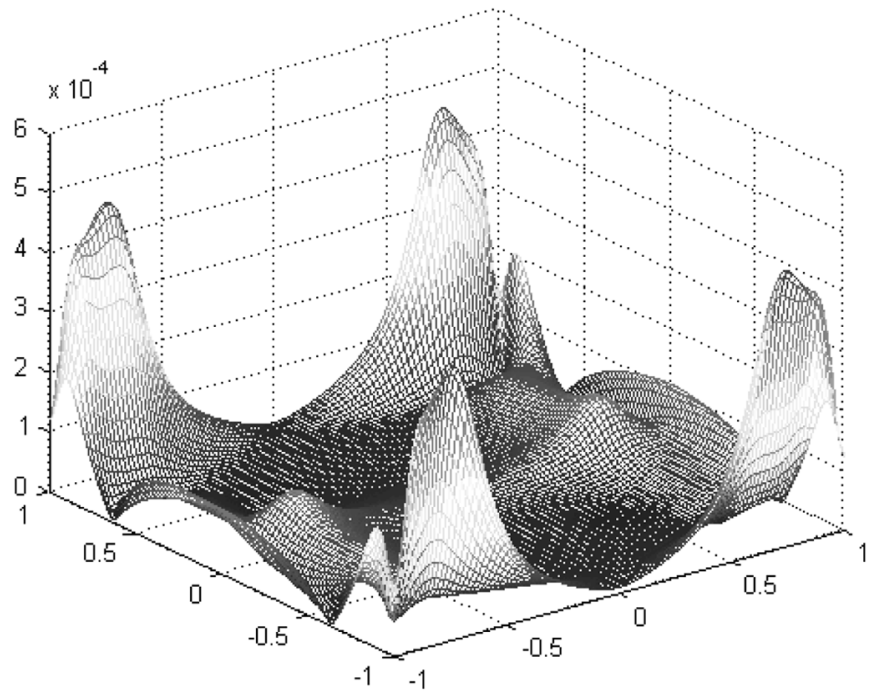

Fig. 6. Reduced-order system.

$$
\begin{aligned}
& A=\left[\begin{array}{cc}
A_{1} & A_{*} \\
0 & A_{2}
\end{array}\right]= \\
& {\left[\begin{array}{cccccc|cccccc}
-0.8827 & 0.2467 & -0.0197 & -0.2019 & -0.0448 & 0.0147 & -0.4224 & -0.1627 & 0.1170 & 0.2617 & -0.0794 & 0.0541 \\
0.1298 & 0.6887 & 0.4814 & 0.2131 & 0.2621 & 0.0536 & -0.0054 & -0.4688 & 0.1862 & -0.1331 & 0.0445 & 0.0116 \\
-0.0160 & -0.4338 & 0.8053 & -0.3793 & -0.0374 & 0.0076 & -0.0915 & -0.0561 & -0.0515 & -0.0676 & -0.0148 & -0.0139 \\
0.1479 & -0.1130 & -0.3140 & -0.5244 & 0.5713 & 0.1098 & 0.1479 & -0.1130 & -0.3140 & -0.5244 & 0.5713 & 0.1098 \\
-0.0877 & -0.1005 & -0.0245 & -0.0376 & -0.0684 & 0.6729 & -0.0877 & -0.1005 & -0.0245 & -0.0376 & -0.0684 & 0.6729 \\
0.0698 & -0.0601 & 0.0085 & 0.2492 & -0.1493 & 0.6582 & -0.2692 & -0.0191 & -0.0885 & 0.1150 & -0.0227 & -0.0201 \\
\hline 0 & 0 & 0 & 0 & 0 & 0 & -0.5743 & 0.1868 & 0.2313 & -0.3841 & 0.0765 & -0.0121 \\
0 & 0 & 0 & 0 & 0 & 0 & -0.6532 & 0.1874 & -0.3837 & 0.1191 & 0.0107 & -0.0187 \\
0 & 0 & 0 & 0 & 0 & 0 & -0.0827 & -0.7194 & -0.5015 & -0.1799 & 0.0444 & 0.0050 \\
0 & 0 & 0 & 0 & 0 & 0 & 0.1685 & 0.3515 & -0.6241 & -0.1327 & 0.1096 & 0.0708 \\
0 & 0 & 0 & 0 & 0 & 0 & 0.0111 & 0.0186 & 0.0134 & -0.4412 & -0.7812 & 0.0168 \\
0 & 0 & 0 & 0 & 0 & 0 & 0.0000 & 0.0041 & -0.0396 & 0.1736 & -0.2118 & -0.9188
\end{array}\right]} \\
& B=\left[\begin{array}{ll}
B_{*} & B_{2}
\end{array}\right]^{T}
\end{aligned}
$$

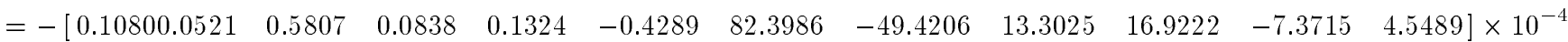

$$
\begin{aligned}
& C=\left[\begin{array}{ll}
C_{1} & C_{*}
\end{array}\right]
\end{aligned}
$$

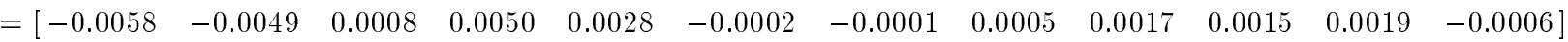


Remark 6: The proposed algorithms are computationally very expensive for large scale systems and are, hence, suitable for only small to medium scale systems. Therefore, computational enhancements such as [11], [12], and [15] proposed for balanced truncation [8] are highly desirable for the proposed algorithms.

\section{CONCLUSION}

An improved frequency interval Gramians based model reduction scheme for discrete time systems is proposed. The algorithms presented for model reduction of 1-D and 2-D discrete systems overcome the shortcomings of the method proposed in [20]. The algorithms presented has two main advantages which include: 1) stability of reduced-order models and 2) easily computable error bounds. Numerical simulation results shows that the proposed algorithms give a better approximation of the original system over a desired frequency interval.

\section{REFERENCES}

[1] P. K. Aghaee, A. Zilouchian, S. N. Ravesh, and A. H. Zadegan, "Principal of frequency domain balanced structure in linear systems and model reduction," Comput. Elec. Eng., vol. 29, pp. 463-477, 2003.

[2] U. M. Al Saggaf and G. F. Franklin, "An error bound for discrete reduced-order model of a linear multivariable system," IEEE Trans. Autom. Control, vol. AC-32, pp. 815-819, 1987.

[3] D. F. Enns, "Model reduction with balanced realizations: An error bound and a frequency weighted generalization," in Proc. 23rd IEEE Conf. Decision Contr., 1984, pp. 127-132.

[4] W. Gawronski and J.-N. Juang, "Model reduction in limited time and frequency intervals," Int. J. Syst. Sci., vol. 21, no. 2, pp. 349-376, 1990.

[5] S. Gugercin and A. C. Antoulas, "A survey of model reduction by balanced truncation and some new results," Int. J. Contr., vol. 77, no. 8, pp. 748-766, 2004.

[6] L. G. Horta, J. N. Juang, and R. W. Longman, "Discrete-time model reduction in limited frequency ranges," J. Guid., Contr. Dynam., vol. 16, no. 6, pp. 1125-1130, 1993.

[7] L. Lerer and A. C. M. Ran, "A new inertia theorem for Stein equations, inertia of invertible hermitian block Toeplitz matrices and matrix orthogonal polynomials," Integr. Eq. Oper. Theory, vol. 47, pp. 339-360, 2003.

[8] B. C. Moore, "Principal component analysis in linear systems: Controllability, observability, and model reduction," IEEE Trans. Autom. Control, vol. AC-26, pp. 17-32, 1981.

[9] N. L. Prajapati, D. Chandra, and D. Seshachalam, "Corrections and comments to ls "Model reduction of discrete linear systems by frequency-domain balanced structure," IEEE Trans. Circuits Syst. I, vol. 54, pp. 682-683.

[10] R. P. Roesser, "A Discrete State Space Model for Linear Image Processing," IEEE Trans. Autom. Control, vol. 20, pp. 1-10, 1975.

[11] M. G. Safonov and R. Y. Chiang, "A Schur method for balancedtruncation model reduction," IEEE Trans. Autom. Control, vol. 34, pp. 729-733, 1989.

[12] M. S. Tombs and I. Postlethwaite, "Truncated balanced realization of a stable non-minimal state-space system," Int. J. Contr., vol. 46, pp. 1319-1330, 1987.
[13] L. Pernebo and L. M. Silverman, "Model reduction via balanced state space representations," IEEE Trans. Autom. Control, vol. AC-27, pp. 382-387, 1982.

[14] R. Treasure, A. Ghafoor, V. Sreeram, and K. Ngan, "Frequency weighted identification and model reduction of 2-D separable denominator systems," in Proc. 6th Int. Conf. Opt. Techn. Appl., Ballarat, Australia, Dec. 9-11, 2004.

[15] A. Varga, A. El Moudni, P. Borne, and S. G. Tzafestas, Eds., "Efficient minimal realization procedure based on balancing," in Proc. IMACS Symp. Modelling Contr. Technol. Syst., Lille, France, 1991, vol. 2, pp. 42-47, Preprints.

[16] A. Varga and B. D. O. Anderson, "Accuracy-enhancing methods for balancing-related frequency-weighted model and controller reduction," Automatica, vol. 39, pp. 919-927, 2003.

[17] D. Wang and A. Zilouchian, "Corrections and comments to 'model reduction of discrete linear systems via frequency-domain balanced structure," IEEE Trans. Circuits Syst. I, vol. 54, pp. 683-684.

[18] G. Wang, V. Sreeram, and W. Q. Liu, "A new frequency-weighted balanced truncation method and an error bound," IEEE Trans. Autom. Control, vol. 44, no. 9, pp. 1734-1737, 1999.

[19] D. Wang, "Identification and approximation of 1-D and 2-D digital filters," Ph.D. dissertation, Florida Atlantic Univ., Boca Raton, FL, 1998.

[20] D. Wang and A. Zilouchian, "Model reduction of discrete linear systems via frequency domain balanced realization," IEEE Trans. Circuits Syst. I, Fund. Theory Appl., vol. 47, no. 6, pp. 830-837, 2000.

[21] K. Zhou, Y. Li, and E. B. Lee, "Model reduction of 2-D systems with frequency error bounds," IEEE Trans. Circuits Syst. II: Analog Digit. Signal Process., vol. 40, no. 2, pp. 107-110, 1993.

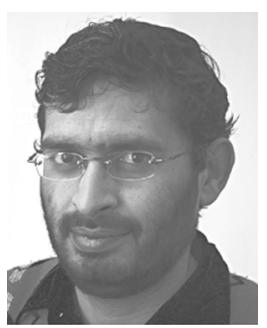

Abdul Ghafoor received the B.S. degree in electrical engineering from the University of Engineering and Technology, Pakistan, in 1994, the M.S. degree in electrical engineering from the National University of Sciences and Technology, Pakistan, in 2003, and the Ph.D. degree from the University of Western Australia.

From 1999 to 2002, he held teaching assignments with the National University of Sciences and Technology. His research topics include model/controllerorder reduction and image processing/matching. Currently, he is with the Military College of Signals, the National University of Sciences and Technology.

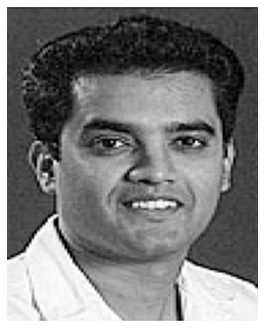

Victor Sreeram (SM'05) received the B.S. degree from Bangalore University, India, in 1981, the M.S. degree from Madras University, India, in 1983, and the Ph.D. degree from the University of Victoria, Canada, in 1989, all in electrical engineering.

He worked as a Project Engineer with the Indian Space Research Organisation from 1983 to 1985 . He joined the Department of Electrical and Electronic Engineering, University of Western Australia, in 1990, where he is now an Associate Professor. He has held Visiting Appointments with the Department of Systems Engineering, Australian National University, during 1994-1996, and with the Australian Telecommunication Research Institute, Curtin University of Technology, during 1997-1998. His research interests are control and signal processing. 